\title{
Evaluación de la calidad de un Manual de Autocuidado para pacientes en Hemodiálisis Crónica
}

\author{
María Isabel Catoni Salamanca**, \#\# \\ Eugenia Eliana Palma Castro*, \#, \#\# \\ Erika Caballero Muñoz**, \#\# \# \\ María Cecilia Arechabala Mantuliz*, \#\# \\ Melan Soledad Peralta Kong**, \# \\ Marisol Rebolledo**, \#\#\#\# \\ Paola de Lourdes Carrasco Aldunate** , \# \#
}

\author{
Escuela de Enfermería de la Universidad Católica. \\ Unidad de Hemodiálisis. Unidad de Diálisis Peritoneal. \\ Hospital Clínico. Universidad Diego Portales. \\ Clínica Alemana. Santiago de Chile \\ * Enfermera \\ ** Enfermera-matrona \\ * Hospital Clínico. Universidad \\ Católica de Chile \\ \#\# Escuela de Enfermería. \\ Universidad Católica de Chile \\ \#\#\# Universidad Diego Portales \\ \#\#\#\# Clínica Alemana
}

\section{RESUMEN}

Estudio descriptivo, transversal, que pretende evaluar la opinión de una población de enfermeras (os) sobre la calidad del Manual de Autocuidado "Aprendiendo a vivir con hemodiálisis" y conocer las características de las actividades educativas realizadas utilizando este Manual como medio instruccional. El instrumento utilizado corresponde a una pauta de evaluación de texto, adaptada de Vergara, Rodríguez y Rittershaussen (1996), la que se aplicó a una muestra al azar, no-probabilística, de 85 enfermeras (os). La validación estadística del instrumento mostró un Alpha de Crombach de 0.65. Los resultados muestran que los tres aspectos evaluados, formato, contenido y aspectos instruccionales, son valorados como ade-

Correspondencia:

María Isabel Catoni Salamanca

P. Universidad Católica de Chile

Campus San Joaquín

Escuela de Enfermería

Avenida Vicuña Mackenna 4860

Santiago

Chile

Mail: <mcatoni@puc.cl> cuados por más del 90\% de los evaluadores. Entre los aspectos que se sugiere mejorar destacan la legibilidad, incorporar estrategias para motivar el aprendizaje en los pacientes e indicar más claramente como utilizar el Manual. El 48,2\% de las enfermeras (os) había realizado actividades educativas utilizando el Manual, la mayoría de ellas eran lectura y discusión de los temas durante la sesión de hemodiálisis.

$\begin{array}{ll}\text { PALABRAS CLAVE: } & \text { EVALUACIÓN } \\ & \text { MATERIAL IMPRESO } \\ & \text { EDUCACIÓN } \\ & \text { AUTOCUIDADO } \\ & \text { HEMODIÁLISIS }\end{array}$

EVALUATION OF THE QUALITY OF A SELF-CARE MANUAL FOR CHRONIC HAEMODYALISIS PATIENTS

This was a descriptive, cross-sectional study evaluating nurses' opinions about the quality of the "Learning to live with Haemodyalisis" Self-Care Manual, and describing the ways in which this 
manual could be used as an eduactional resource. The instrument that was used was adpated from the text evaluation guidelines developed by Vergara, Rodriguez, and Rittershaussen (1996). The non-probability sample included 85 nurses. The statistical analysis of the data indicated that the instrument had a Crombach alpha internal consistency reliability of 0.65 . The results indicated that $90 \%$ of the nurses in the sample evaluated the format, content, and instructional aspects of the manual as appropriate. The nurses recommended several improvements in the manual, including enhacing the legibility, incorporating more strategies to motivate patient learning, and to clarify how to use a manual. A total of $48.2 \%$ of the nurses in the sample had used the Manual as a resource for patient education. The most common educational activities developed using the manual were reading and discussion with patients during their haemodyalisis sessions.

\section{KEY WORDS: EVALUATION}

\section{PRINTED MATERIAL \\ HEALTH EDUCATION \\ SELF-CARE \\ HAEMODYALISIS}

El material impreso ha sido extensamente utilizado en la educación de pacientes en diferentes áreas, principalmente con el objeto de que los usuarios comprendan su enfermedad, aprendan sobre como cuidarse, puedan tomar decisiones informadas con respecto a su propio cuidado o acepten y cumplan el tratamiento ${ }^{(1,2,3,4)}$. El último componente del proceso educativo, la evaluación, es el paso que con mayor frecuencia se omite en este tipo de programas, siendo una etapa muy necesaria porque es en ella donde finalmente se determina el éxito o falla del material o del programa ${ }^{(5)}$. Se han utilizado diferentes instrumentos para evaluar material educativo impreso, ya sea con el fin de determinar la adecuación del material al nivel de comprensión de lectura del usuario ${ }^{(6,7,7,9)}$, su impacto sobre los conocimientos, actitudes y conducta del paciente $e^{(2,4,10,11,12)}$ o su calidad en general ${ }^{(13,18)}$. Vergara et al. crearon y validaron un instrumento para evaluar textos de aprendizaje, utilizando a los educadores como evaluadores. El instrumento de Vergara destaca por su integralidad, ya que considera tanto aspectos del formato y del contenido, como aspectos instruccionales ${ }^{(18)}$. El presente trabajo tiene como propósito evaluar un Manual de Autocuidado para pacientes en $\mathrm{He}$ - modiálisis Crónica (HDC), que fue distribuido a los enfermeros (as) que trabajan en Centros de Hemodiálisis en la Ciudad de Santiago de Chile, en el primer semestre de 2001.

\section{DESCRIPCIÓN DEL MANUAL DE AUTOCUIDADO "APRENDIENDO A VIVIR CON HEMODIÁLISIS"}

El manual consta de 179 páginas en un tamaño de 20 x $15 \mathrm{~cm}$ (Fig. 1). Está escrito en una columna e incluye ilustraciones, tablas, esquemas y cuadros resúmenes, que destacan los aspectos relevantes. Cada tema se introduce con una pregunta con el fin de motivar el aprendizaje. Contiene una introducción, diez capítulos y una sección de anexos, ordenados de la siguiente manera: 1) Insuficiencia renal crónica y alternativas de tratamiento. 2) Indicaciones para sus primeras hemodiálisis. 3) Cómo enfrentar una enfermedad crónica. 4) Cuidados del acceso vascular. 5) Presión arterial y hemodiálisis. 6) Nutrición y ejercicio. 7) Manejo del calcio y del fósforo. 8) Anemia y hemodiálisis. 9) Diabetes y hemodiálisis. 10) Beneficios de salud y previsión. 11) Anexos: Minuta semanal y Tablas de composición química de los alimentos (Disponible en Internet en <www.senferdialt.cl>).

\section{Aprendienclo \\ al vivir cori \\ Herrodliélisis}

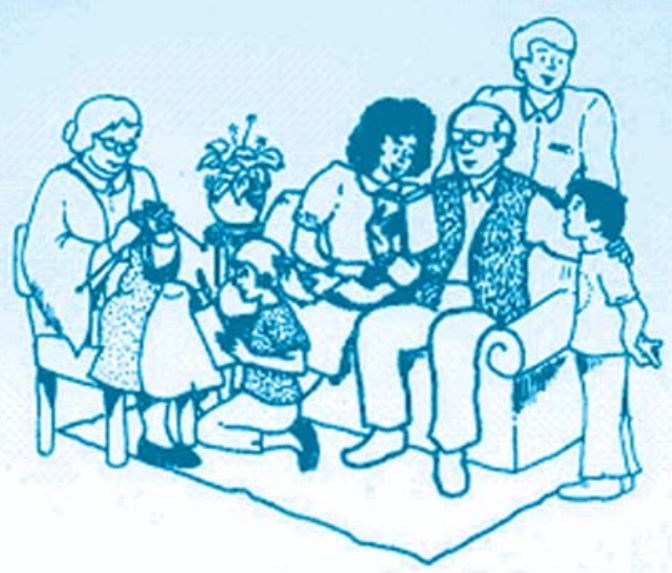

Manual de autocuidado para el paciente en hemodiálisis

Figura 1. Manual de autocuidado. 


\section{MATERIAL Y MÉTODO}

Para seleccionar la muestra se utilizó la lista actualizada de Centros de Diálisis de la Asociación de Dializados de Chile (ASODI), institución que se encargó de distribuir el Manual antes de este estudio. Se llamó por teléfono a todos los Centros de Diálisis situados en la ciudad de Santiago, consultando si habían recibido el Manual y solicitando el consentimiento para realizar una encuesta. A los Centros que contestaron afirmativamente se les solicitó una hora para realizar la encuesta y el día acordado se entregó el instrumento a los enfermeros (as) que estaban de turno y que aceptaron participar en el estudio. Las encuestas, una vez respondidas, se retiraron el mismo día. De este modo, la muestra quedó conformada por un total de 85 enfermeros (as), que trabajaban en 37 Centros de Diálisis.

El instrumento utilizado corresponde a la pauta de evaluación de texto, adaptada de Vergara, Rodríguez y Rittershaussen ${ }^{(18)}$, la que evalúa básicamente tres dimensiones: aspectos formales, aspectos del contenido y aspectos instruccionales, los que a su vez se desglosan en 29 ítems que el encuestado debe contestar según una escala de Likert, que va desde "muy de acuerdo" hasta "muy en desacuerdo", más la categoría "no observable". Al concluir la evaluación de cada dimensión, se solicita una evaluación global de la misma, con su respectiva fundamentación. Para el análisis de los resultados se agruparon las respuestas "muy de acuerdo" y "de acuerdo", considerando que para estas personas el manual tenía un nivel "adecuado" en esos ítems; por el contrario las respuestas "en desacuerdo" y "muy en desacuerdo" se agruparon considerando que para las personas el manual tenía un nivel "inadecuado" en esos ítems. Con los datos obtenidos en esta muestra se procedió a validar estadísticamente el instrumento, obteniéndose un Alpha de Crombach de 0.65. El análisis de los datos se realizó con el programa estadístico SPSS.

\section{RESULTADOS}

1.- Características de los enfermeros (as): $\mathrm{El}$ 95,3\% de la muestra era de sexo femenino. Los enfermeros (as) llevaban trabajando en HDC en promedio 8,9 años. Al momento del estudio cada uno había tenido en su poder en promedio, 4 meses el manual. El 100\% declara haber leído el manual (55,3\% en forma parcial, 44,7\% completamente).

\section{2.- Actividades educativas en las que se utilizó el} manual: El 48,2\% de los enfermeros (as) había realizado actividades educativas en las que utilizaba el manual. La mayoría señaló que realizaba educación durante el procedimiento de diálisis, entregando el manual a los pacientes, para luego analizar los temas en conjunto y solucionar dudas. Algunos enfermeros (as) citaban al usuario y familia en horario de no-diálisis. Otros educaron sólo a los pacientes nuevos. Los capítulos más utilizados fueron los de alimentación y aspectos psicosociales. Llama la atención que los enfermeros (as) refieren haberlo usado también en la formación de otros enfermeros (as) y de personal auxiliar.

\section{3.- Evaluación de aspectos formales del manual:}

En promedio el porcentaje de logro de los indicadores es bastante alto, 94,8\% en formato, 96,8\% en legibilidad y $92 \%$ en uso del color e imágenes. Los ítems con más baja evaluación son la encuadernación, el uso del color y la cantidad de ilustraciones, tablas y fotos (Tabla 1).

\begin{tabular}{|c|c|c|c|}
\hline Dimensión & Indicador & Adecuado & Inadecuado \\
\hline Formato & $\begin{array}{l}\text { Encuadernación } \\
\text { Calidad del papel } \\
\text { Tamaño } \\
\text { Promedio de logro en formato }\end{array}$ & $\begin{array}{l}89,3 \% \\
97,6 \% \\
97,6 \% \\
94,8 \%\end{array}$ & $\begin{array}{l}10,7 \% \\
2,4 \% \\
2,4 \% \\
5,2 \%\end{array}$ \\
\hline Legibilidad & $\begin{array}{l}\text { Construcción gramatical } \\
\text { Vocabulario y expresiones } \\
\text { Tamaño y tipo de letra } \\
\text { Contraste entre letras y fondo } \\
\text { Distancia entre palabras } \\
\text { Espacios entre líneas y párrafos } \\
\text { Uso de márgenes } \\
\text { Uso de columnas de lectura } \\
\text { Proporción de texto e imágenes } \\
\text { Promedio de logro en legibilidad }\end{array}$ & $\begin{array}{l}97,6 \% \\
97,6 \% \\
97,6 \% \\
97,6 \% \\
97,6 \% \\
92,9 \% \\
99,0 \% \\
97,6 \% \\
94,1 \% \\
96,8 \%\end{array}$ & $\begin{array}{l}2,4 \% \\
2,4 \% \\
2,4 \% \\
2,4 \% \\
2,4 \% \\
7,1 \% \\
1,0 \% \\
2,4 \% \\
5,9 \% \\
3,2 \%\end{array}$ \\
\hline $\begin{array}{l}\text { Uso de } \\
\text { color e } \\
\text { imágenes }\end{array}$ & $\begin{array}{l}\text { Uso del color } \\
\text { Cantidad de ilustraciones, tablas } \\
\text { y fotos, etc } \\
\text { Nitidez de la imágenes } \\
\text { Tamaño de las imágenes } \\
\text { Atingencia de ilustraciones, tablas } \\
\text { y fotos } \\
\text { Ilustraciones complementan el } \\
\text { texto escrito } \\
\text { Promedio de logro en color e imag }\end{array}$ & $\begin{array}{l}78,1 \% \\
83,4 \% \\
98,8 \% \\
96,5 \% \\
97,7 \% \\
97,6 \% \\
92,0 \%\end{array}$ & $\begin{array}{c}21,9 \% \\
16,6 \% \\
1,2 \% \\
3,5 \% \\
2,3 \% \\
2,4 \% \\
8,0 \%\end{array}$ \\
\hline
\end{tabular}

Tabla 1. Evaluación aspectos formales. 
La evaluación global de los aspectos formales alcanza un $78,8 \%$ de logro de los indicadores. Al fundamentar su opinión los enfermeros (as) que evalúan como "adecuados" los aspectos formales, señalan que el texto es adecuado en tamaño y legibilidad, de ideas claras y precisas, sintético, de presentación agradable, lenguaje amigable, completo, ameno y didáctico. Por el contrario, los enfermeros (as) que evaluaron los aspectos formales como "medianamente adecuados", opinan que sería mejor un formato más pequeño, parcelarían más los contenidos, agregarían ilustraciones que complementen el texto escrito y más color para destacar aspectos relevantes. Algunos enfermeros (as) señalan que sería conveniente aumentar el tamaño de las tablas, de las imágenes, los espacios entre frases y párrafos y el contraste con el fondo, debido a que una parte importante de los pacientes presenta problemas visuales. Algunos evaluadores señalan que el manual debiera contar con más aspectos gráficos y menos lectura, dado el bajo nivel educacional de la mayoría de los pacientes. Dos enfermeros (as) consideran que el lenguaje es "elevado" para los pacientes, pero es muy útil para el personal de salud. Por último, es importante destacar que ningún enfermero (a) evaluó los aspectos formales como inadecuados.

4.- Evaluación de aspectos del contenido: En promedio el porcentaje de logro de los indicadores de pertinencia del contenido es de 96,9\%, en calidad del contenido es de $93,1 \%$ y en organización del mismo es de $87,1 \%$. Los ítems peor evaluados son la introducción, el índice, las tablas y el propósito (Tabla 2).

En la percepción global de los enfermeros (as) con respecto a la selección y presentación de los contenidos, evaluados en un rango de "contenido no pertinente" (0 puntos) a "contenido pertinente" (5 puntos), un $82,3 \%$ considera los contenidos pertinentes (4-5 puntos). Al fundamentar su apreciación los enfermeros (as) que consideran los contenidos pertinentes, señalan que estos son precisos, didácticos, adecuados y de interés para los pacientes y sus familias, abarcando los temas de mayor importancia en forma práctica, clara y precisa. También destacan que los resúmenes refuerzan lo más importante del capítulo y que los temas son abordados en forma integral.

Las personas que consideraron los contenidos del texto como medianamente pertinentes, señalan que está muy dirigido a los cristianos, lo que a su juicio introduce un

\begin{tabular}{|c|c|c|c|}
\hline Dimensión & Indicador & Adecuado & Inadecuado \\
\hline $\begin{array}{l}\text { Pertinencia } \\
\text { de los } \\
\text { contenidos }\end{array}$ & $\begin{array}{l}\text { Adecuado a los usuarios } \\
\text { Satisface los requerimientos } \\
\text { de los pacientes } \\
\text { Propuesta valórica es acorde } \\
\text { a la cultura nacional } \\
\text { Propuesta valórica es acorde } \\
\text { a los principios de la PUC } \\
\text { Promedio de logro en } \\
\text { pertinencia }\end{array}$ & $\begin{array}{l}97,7 \% \\
98,8 \% \\
92,4 \% \\
98,8 \% \\
96,92 \%\end{array}$ & $\begin{array}{r}2,3 \% \\
1,2 \% \\
7,6 \% \\
1,2 \%\end{array}$ \\
\hline $\begin{array}{l}\text { Calidad } \\
\text { de los } \\
\text { contenidos }\end{array}$ & $\begin{array}{l}\text { Exactitud del contenido } \\
\text { Profundidad del contenido } \\
\text { Presencia de sesgos } \\
\text { ideológicos } \\
\text { Promedio de logro en } \\
\text { calidad }\end{array}$ & $\begin{array}{l}96,1 \% \\
92,9 \% \\
90,3 \% \\
93,1 \%\end{array}$ & $\begin{array}{l}3,9 \% \\
7,1 \% \\
9,7 \%\end{array}$ \\
\hline $\begin{array}{l}\text { Organización } \\
\text { de los } \\
\text { contenidos }\end{array}$ & $\begin{array}{l}\text { Introducción facilita el } \\
\text { aprendizaje } \\
\text { Indice y tablas facilitan el } \\
\text { aprendizaje } \\
\text { Unidades o capítulos } \\
\text { facilitan el aprendizaje } \\
\text { Propósito facilita el } \\
\text { aprendizaje } \\
\text { Promedio de logro en } \\
\text { organización }\end{array}$ & $\begin{array}{l}76,2 \% \\
88,1 \% \\
96,5 \% \\
87,4 \% \\
87,15 \%\end{array}$ & $\begin{array}{l}23,8 \% \\
11,9 \% \\
3,5 \% \\
12,6 \%\end{array}$ \\
\hline
\end{tabular}

Tabla 2. Evaluación aspectos del contenido.

sesgo, sugieren que el capítulo sobre "como enfrentar una enfermedad crónica" se enfoque desde una postura más abierta. Indican que es necesario profundizar los temas: anemia, trasplante y nutrición. Algunos enfermeros (as) señalan que si bien los contenidos son pertinentes, el vocabulario no es de fácil comprensión para la mayoría de los pacientes.

\section{5.- Evaluación de aspectos instruccionales: El por-} centaje de logro de la función facilitadora del aprendizaje es de $89.97 \%$ y el de la organización de los componentes instruccionales es de 93,55\%. Los ítems peor evaluados son la introducción, la ilustración de los conceptos relevantes con ejemplos y el uso de tipografía, destacadores y recuadros (Tabla 3).

Al consultar la opinión global de los enfermeros (as), respecto a la organización de los componentes instruccionales como facilitadores del aprendizaje, un 78,9\% de los encuestados señalan que estos contribuyen y un $21,2 \%$ que contribuyen medianamente. Los enferme- 


\begin{tabular}{|c|c|c|c|c|}
\hline Dimensión & Subdimensión & Indicador & Adecuado & Inadecuado \\
\hline $\begin{array}{l}\text { Función } \\
\text { facilitadora } \\
\text { del } \\
\text { aprendizaje }\end{array}$ & $\begin{array}{l}\text { Motivación } \\
\text { Comprensión } \\
\text { Relevancia } \\
\text { Refuerzo de } \\
\text { información }\end{array}$ & $\begin{array}{l}\text { Introducción tiene } \\
\text { sentido } \\
\text { motivador } \\
\text { Presentación facilita } \\
\text { la comprensión } \\
\text { Se ilustran los conceptos } \\
\text { relevantes } \\
\text { Resúmenes y síntesis } \\
\text { refuerzan la información } \\
\text { relevantes } \\
\\
\text { Promedio de logro en } \\
\text { f. facilitadora del } \\
\text { aprendiz. }\end{array}$ & $\begin{array}{l}78,8 \% \\
97,6 \% \\
84,7 \% \\
98,8 \%\end{array}$ & $\begin{array}{l}21,2 \% \\
2,4 \% \\
15,3 \%\end{array}$ \\
\hline $\begin{array}{l}\text { Organización } \\
\text { de los } \\
\text { componentes } \\
\text { instruccionales }\end{array}$ & $\begin{array}{l}\text { Adecuación } \\
\text { a las } \\
\text { características } \\
\text { del usuario }\end{array}$ & $\begin{array}{l}\text { Presentación } \\
\text { Objetivos } \\
\text { Esquemas o cuadros } \\
\text { sinópticos } \\
\text { Uso de tipografía, desta- } \\
\text { cadores, recuadros,etc. } \\
\text { Ejemplos e ilustraciones } \\
\text { Resúmenes y síntesis } \\
\text { Promedio de logro en } \\
\text { organización }\end{array}$ & $\begin{array}{l}93,9 \% \\
97,6 \% \\
\\
90,1 \% \\
\\
89,3 \% \\
95,1 \% \\
95 ; 3 \%\end{array}$ & $\begin{array}{c}6,1 \% \\
2,4 \% \\
\\
9,9 \% \\
10,7 \% \\
4,9 \% \\
4,7 \%\end{array}$ \\
\hline
\end{tabular}

Tabla 3. Evaluación aspectos instruccionales.

ros (as) que consideran que los aspectos instruccionales contribuyen al aprendizaje, señalan que es una buena guía para las enfermeras (os), facilita la tarea educativa mostrando los contenidos en forma clara, con una secuencia lógica, motiva a profesionales y pacientes a la lectura. Se destacan los datos prácticos (uso de tablas nutricionales, minutas), también señalan que el uso de los resúmenes, recuadros de color y dibujos sintetizan la información relevante, facilitando el aprendizaje. Las enfermeras (os) consideran que el contenido esta bien enfocado ya que permite al paciente conocer más sobre su enfermedad, estimula la adherencia al tratamiento y la cooperación del paciente en sus cuidados, explicando los peligros a que se expone si no se cuida y promoviendo el apoyo familiar. Los profesionales destacaron la buena acogida que el manual tuvo en los familiares de los usuarios y el hecho de que los pacientes se motivaron por aprender y compartir lo que aprendieron.

Las personas que opinaron que los aspectos instruccionales del manual contribuyeron medianamente a facilitar el aprendizaje, consideran que al manual le faltan nociones básicas respecto a técnicas educativas que permitan motivar el aprendizaje y enseñar a aprender, de hecho indican que "falta una orientación a los profesionales sobre como enseñar". Señalan que el lenguaje es para un nivel educacional alto y que especialmente algunos temas están tratados en un nivel complejo para personas con escolaridad básica y media. Sugieren entregar la información a un nivel más básico y directo, agregar un glosario y aumentar el número de ilustraciones. Con relación a los temas tratados, sugieren adaptar las minutas a las características socioeconómicas del paciente y ampliar la información sobre trasplante y diálisis peritoneal.

\section{CONCLUSIONES Y DISCUSIÓN}

Se concluye que en general el Manual ha sido bien evaluado, la mayoría de los ítems son valorados como adecuados por el $90 \%$ o más de los evaluadores; sin embargo, hay varios aspectos susceptibles de ser mejorados. Con respecto al formato, es necesario hacer un uso más profuso del color y de las imágenes, lo que contribuye a la comprensión del texto y motiva la lectura $^{(14,15,16)}$. En relación con la legibilidad, destaca la constante referencia de los evaluadores al lenguaje, el que consideran de un nivel superior al de la mayoría de los pacientes. Lograr un lenguaje adecuado, quizás sea uno de los puntos más difíciles de lograr en un material impreso ${ }^{(7,8)}$, considerando el nivel educacional de los lectores (el que los evaluadores definen como bajo) y el hecho de que para comprender la terapia necesariamente deben incorporar conceptos complejos. Una alternativa que puede facilitar la comprensión del manual, es la propuesta realizada por algunos evaluadores de agregar un glosario al final del texto. Horner en este sentido recomienda incluir tanto la definición de términos complejos, como de los que pudieran ser nuevos (14). Otro aspecto que contribuye a la legibilidad y que puede ser fácilmente mejorado, es el que se refiere a aumentar el tamaño de letras y números, sobretodo en las tablas de composición química de los alimentos, considerando el deterioro de la visión propio de los adultos mayores y de la presencia de diabetes e hipertensión de larga data en muchos pacientes. El tamaño de letra utilizado en el Manual es 12, en algunos recuadros 14 , las tablas y minutas están en tamaño 10 . El tamaño de letra recomendado es mínimo 12 a 14 e idealmente $18^{(14)}$.

En relación con los aspectos del contenido, los evaluadores indican que el manual no evidencia problemas a nivel de pertinencia y calidad del contenido. Algunos sugieren profundizar en los temas de trasplante, diálisis peritoneal, anemia y nutrición. Con respecto a trasplante y diálisis peritoneal, es necesario considerar que 
el objetivo del Manual, tal como lo indica su nombre, es aprender a vivir con hemodiálisis.

La introducción es el ítem peor evaluado, de acuerdo a los resultados le faltan elementos que motiven a la lectura y que faciliten el aprendizaje. Por otra parte, algunos evaluadores consideran que la explicitación del propósito del Manual es inadecuada y que no contiene elementos instruccionales que guíen a los enfermeros (as), sobre como utilizarlo. A este respecto es necesario recordar que este Manual fue escrito pensando en que los enfermeros (as) lo utilizaran como guía en la educación para el autocuidado que habitualmente realizan a los pacientes, teniendo presente que la información escrita no puede ser el único medio para educar al paciente ${ }^{(19)}$. En relación al mismo punto, Mayeaux et al. plantean la necesidad de combinar la instrucción verbal y escrita para mejorar la comprensión y adherencia del paciente al plan de cuidados ${ }^{(16)}$. Por otra parte, dado que el material impreso debe ser necesariamente igual para todos los pacientes, la comunicación verbal puede cumplir el rol de contener elementos que hagan los contenidos más consistentes con el nivel de comprensión y experiencias del paciente ${ }^{(15)}$. Ninguno de estos aspectos estaba explicitado en el Manual, de modo que en una próxima edición es necesario incluir una exposición breve sobre como utilizarlo.

A juicio de los evaluadores, el Manual tiene un sesgo ideológico, interpreta la espiritualidad sólo desde el punto de vista de los cristianos, aspecto que es congruente con la visión de la Pontificia Universidad Católica de Chile, institución que patrocina el Manual, y con la cultura nacional. De hecho en Chile el 70\% de los ciudadanos de 15 años y más, se declara de religión católica ${ }^{(20)}$; sin embargo, lógicamente hay un porcentaje que no se siente identificado con esta postura. La inclusión de aspectos congruentes con la cultura de los usuarios es relevante, lo contrario podría generar un rechazo del contenido del material educativo en general ${ }^{(16,17)}$. En una próxima edición es importante buscar estrategias para resolver este aspecto.

\section{BIBLIOGRAFÍA}

1. Johnson LK, Edelman A, Jensen J. Patient satisfaction and the impact of written material about postpartum contraceptive decisions. Am J Obstet Gynecol 2003; May 188(5):1202-4.
2. Milewa T, Calnan M, Almond S, Hunter A. Patient education literature and help seeking behaviour: perspectives from an evaluation in the United Kingdom. Soc Sci Med 2000; Aug 51(3): 463-75.

3. Piccoli GB, Mezza E, Iadarola AM, Bechis F, Anania P, Vischi M, lacuzzo C, Gai M, Martino B, Garofletti Y, Giraudo G, Jeantet A, Segoloni GP. Education as a clinical tool for self-dialysis. Adv Perit Dial 2000;16: 186-90.

4. Schlatter S, Ferrans C.E. Teaching program effects on high phosphorus levels in patients receiving hemodialysis. ANNA J 1998; Feb 25(1):31-8.

5. Frank-Stromborg M, Cohen R. Evaluating written patient education materials. Semin Oncol Nurs 1991; May 7(2):125-34.

6. French K. S, Larrabee J.H. Relationships among educational material readability, client literacy, perceived beneficence, and perceived quality. J Nurs Care Qual 1999; Aug 13(6):68-82.

7. Fisher E. Low literacy levels in adults: implications for patient education. J Contin Educ Nurs 1999; MarApr 30(2):56-61.

8. Dowe M.C, Lawrence P.A, Carlson J, Keyserling T.C. Patients' use of health-teaching materials at three readability levels. Appl Nurs Res 1997; May 10(2):86-93.

9. Watkins G.R. Patient comprension of gastroenterology (GI) educational materials. Gastroenterol Nurs 1995; Jul-Aug 18 (4):123-7.

10. Charnock D, Shepperd S, Needham G, Gann R. DISCERN: an instrument for judging the quality of written consumer health information on treatment choices. J Epidemiol Community Health 1999; Feb 53 (2):105-11.

11. Pacheco D, Berdichevsky, Ballesteros F, Fuentealba $\mathrm{C}$, Arinoviche, Pérez J. Efectos de un manual educativo sobre conocimientos y actitudes en pacientes reumáticos y personal de salud. Revista Médica de Chile 1996; 124:1071-1076.

12. Higgins L, Ambrose P. The effect of adjunct questions on older adults' recall of information from a patient education booklet. Patient Educ Couns 1995; Feb 25 (1):67-74.

13. Farrell-Miller P, Gentry P. How effective are your patient education materials? Guidelines for developing and evaluating written educational materials. Diabetes Educ 1989; Sep-Oct 15(5):418-22.

14. Horner S.D, Surratt D, Juliusson S. Improving readability of patient education materials. Journal of community health nursing 2000; 17 (1):15-23. 
15. Brooks D. A. Techniques for teaching ED patients with low literacy skills. Journal of Emergency Nursing 1998; 24:601-603.

16. Mayeaux E.J, Murphy P.W, Arnold C, Davis T.C, Jackson R.H. and Sentell T. Improving patient education for patients with low literacy skills. American Family Physician 1996; 53:205-211.

17. Vahabi M. and Ferris L. Improving written patient education materials: A review of the evidence. Health Education Journal 1995; 54: 99-106.

18. Vergara A, Rodríguez E, Rittershaussen S. Un texto que permita aprender. Boletín de Investigación
Educacional. Facultad de Educación. Santiago 1996; 11: 218-235.

19. Williams M.V, Baker D.W, Parker R.M. and Nurss J.R. Relationship of functional health literacy to patients knowledge of their chronic disease: A study of patients with hypertension and diabetes. Archives of Internal Medicine 1998; 158:166-172.

20. INE (Instituto Nacional de Estadísticas), Censo 2002. Síntesis de resultados. Santiago, Chile. Pag 25. 